\title{
Nivolumab for the Treatment of Esophageal Squamous Cell Carcinoma
}

\author{
Mao Okada, Shun Yamamoto, and Ken Kato \\ Department of Head and Neck Medical Oncology, National Cancer Center Hospital, Tokyo, Japan
}

DOI: https://doi.org/10.17925/OHR.2021.16.2.90

E sophageal cancer is the seventh most common malignancy, for which there are few effective treatment options, especially for patients with advanced disease. Therefore, the therapeutic development of immune checkpoint inhibitors has been the focus for patients with advanced esophageal cancer. Nivolumab, a human monoclonal immunoglobulin G4 antibody that inhibits programmed cell death protein 1, has been developed for this patient population. The phase II ATTRACTION-1 trial showed promising efficacy for nivolumab in patients with advanced esophageal cancer who were refractory or intolerant to fluoropyrimidine-, platinum-, and taxane-based chemotherapies. The phase III ATTRACTION-3 trial showed the superiority of nivolumab over taxane in patients with advanced esophageal squamous cell carcinoma (ESCC) who were refractory or intolerant to previous fluoropyrimidine- and platinum-based chemotherapy. Based on these results, the FDA approved nivolumab as a treatment for patients with advanced ESCC after prior fluoropyrimidine- and platinum-based chemotherapy. Additionally, the CheckMate 577 trial has shown that nivolumab improves disease-free survival when used as an adjuvant treatment in patients with resectable tumors who received neoadjuvant chemoradiation followed by surgery, and in whom a pathological complete response was not achieved. Clinical trials to evaluate the efficacy of nivolumab in combination with cytotoxic drugs as first-line chemotherapy, in combination with chemoradiotherapy, or for the treatment of perioperative patients with esophageal cancer are ongoing and might contribute to the establishment of new standard treatments.

\section{Keywords}

Esophageal adenocarcinoma, esophageal cancer, esophageal squamous cell carcinoma, immune checkpoint inhibitor, nivolumab, PD-1 antibody, pembrolizumab

Disclosures: Shun Yamamoto has received personal fees from ONO Pharmaceuticals. Ken Kato has received research funds from ONO Pharmaceuticals, MSD, Shionogi, Oncolys Biopharma, Chugai, AstraZeneca, and Beigene; and personal fees from Eli Lilly and Taiho. Mao Okada has no financial or non-financial relationships or activities to declare in relation to this article.

Peer Review: Double-blind peer review.

Compliance with Ethics: This study involves a review of the literature and did not involve any studies with human or animal subjects performed by any of the authors.

Authorship: The named authors meet the International Committee of Medical Journal Editors (ICMJE) criteria for authorship of this manuscript, take responsibility for the integrity of the work as a whole, and have given final approval for the version to be published.

Access: This article is freely accessible at touchONCOLOGY.com (c) Touch Medical Media 2020.

Received: October 3, 2020

Accepted: December 15, 2020

Published Online: February 8, 2021

Citation: Oncology \& Hematology Review. 2020;16(2):90-4

Corresponding Author: Ken Kato, Department

of Head and Neck Medical Oncology, Nationa

Cancer Center Hospital, 5-1-1 Tsukiji, Chuo-ku,

Tokyo 104-0045, Japan. E: kenkato@ncc.go.jp

Support: No funding was received in

the publication of this article.
Esophageal cancer is the seventh most common malignancy and the sixth leading cause of death from cancer worldwide. ${ }^{1}$ The main histological subtypes are esophageal squamous cell carcinoma (ESCC) and esophageal adenocarcinoma. ESCC is the most common subtype, and the frequency of this disease is highest in Eastern Asia and Eastern Africa. ${ }^{2}$ In Western countries, however, the incidence of esophageal adenocarcinoma is higher than that of ESCC. ${ }^{2-5}$ The key risk factors associated with ESCC are smoking and alcohol consumption, while the key risk factors of esophageal adenocarcinoma are gastroesophageal reflux disease, obesity, and Barrett esophagus.,

Patients with early-stage esophageal cancer are often asymptomatic, but the majority of patients are diagnosed with advanced disease and have a poor prognosis. ${ }^{5,6}$ In addition, there are few treatment options for metastatic or recurrent patients with esophageal cancer. Doublet chemotherapy, consisting of fluoropyrimidine and platinum, is recognized as the first-line chemotherapy, ${ }^{7,8}$ and taxane or irinotecan monotherapy was used as salvage-line chemotherapy before the development of immune checkpoint inhibitors. ${ }^{9-11}$ No targeted agents have been developed for esophageal cancer, and this disease has been understudied in clinical trials over the past several decades.

\section{Introduction to nivolumab}

Immune checkpoint inhibitors, such as anti-programmed cell death protein 1 (PD-1) antibody, were first used in esophageal cancer to treat metastatic or recurrent disease. PD-1 is an immune checkpoint receptor that is mainly expressed on cytotoxic T cells. The two ligands for PD-1, programmed death ligand 1 (PD-L1) and PD-L2, are expressed on the surface of cancer cells and immune cells in the tumor microenvironment. In the tumor microenvironment, the binding of these ligands to PD-1 suppresses the activation of cytotoxic T cells, which lose their ability to infiltrate the tumor and kill cancerous cells. The fully-humanized immunoglobulin G (IgG)4 monoclonal PD-1 antibody, nivolumab, binds to PD-1 on cytotoxic T cells and inhibits its interaction with the tumor cell PD-L1 and PD-L2 ligands, thereby blocking the transmission of immunosuppressive signals and maintaining T-cell activation, resulting in an anti-tumor effect. ${ }^{12-15}$ Nivolumab is effective in a variety of cancer types-the US Food and Drug Administration (FDA) have approved nivolumab for the treatment of advanced melanoma, advanced non-small cell lung cancer, advanced small cell lung cancer, classical Hodgkin lymphoma, advanced renal cell carcinoma, urothelial carcinoma, squamous cell carcinoma of the head and neck, microsatellite instability-high or mismatch repair-deficient metastatic colorectal cancer, and hepatocellular carcinoma. ${ }^{16-26}$ 
In some clinical trials of immune checkpoint inhibitors, PD-L1 is reported as the candidate of predictive biomarker. Previous reports revealed that PD-L1 overexpression was found in approximately $15-83 \%$ of patients with ESCC, and high PD-L1 expression was associated with poor overall survival (OS). ${ }^{27,28}$ To evaluate PD-L1 expression, tumor proportion score (TPS) or combined positive score (CPS) are often used in clinical trials. TPS is defined as the number of PD-L1 positive tumor cells divided by the total number of tumor cells, multiplied by 100 . On the other hand, CPS is defined as the number of PD-L1 positive tumor cells, macrophages, and lymphocytes divided by the total number of tumor cells, multiplied by 100.

The PD-L1 IHC 28-8 pharmDx assay (Dako, an Agilent Technologies, Inc. company, Santa Clara, CA, USA) is mainly used for evaluating TPS in clinical trials of nivolumab, while PD-L1 IHC 22C3 pharmDx assay (Agilent Technologies, Carpinteria, CA, USA) is mainly used for evaluating CPS is mainly used in clinical trials of pembrolizumab. PD-L1 expression (TPS) evaluated by 28-8 and 22C3 has shown concordance for patients with lung cancer in the BluePrint project; 29 however, there has been no evidence of concordance between TPS and CPS, 28-8 and 22C3 for patients with esophageal cancer.

\section{Nivolumab monotherapy as second-line treatment for patients with advanced esophageal squamous cell carcinoma}

Nivolumab has been developed for the treatment of esophageal cancer, as well as other cancers. The phase II ATTRACTION-1 trial was a singlearm study that evaluated the efficacy of nivolumab monotherapy $(3 \mathrm{mg} / \mathrm{kg}$ every 2 weeks) in patients with metastatic or recurrent ESCC, esophageal adenocarcinoma, or adenosquamous-cell carcinoma of the esophagus who were refractory or intolerant to fluoropyrimidine-based, platinum-based, and taxane-based chemotherapy. Although, ESCC, adeno, and adenosquamous cell patients were eligible to take part in this trial, all 65 patients enrolled in this trial had ESCC as a result (Table 1). ${ }^{30-32}$ Central reviewed response rate of nivolumab was $17 \%$ ( $95 \%$ confidence interval [CI] 10-28), median OS was 10.78 months (95\% $\mathrm{Cl} 7.4-13.3$ ), and median progression-free survival (PFS) was 1.5 months $(95 \% \mathrm{Cl} 1.4-2.8)$. The most frequent treatment-related adverse events of grade 3 or greater were decreased appetite (3\%), lung infection (3\%), increased blood creatinine phosphokinase (3\%), and dehydration (3\%); there were no treatment-related deaths. The ATTRACTION-1 trial showed that nivolumab monotherapy had promising efficacy and induced manageable adverse events in patients with metastatic or recurrent ESCC. ${ }^{30}$

The phase III ATTRACTION-3 trial was an open-label, multicenter study comparing nivolumab monotherapy (240 mg every 2 weeks) with chemotherapy (paclitaxel $100 \mathrm{mg} / \mathrm{m}^{2}$ once per week for 6 weeks then 1 week off, or docetaxel $75 \mathrm{mg} / \mathrm{m}^{2}$ every 3 weeks) for ESCC or adenosquamous carcinoma. ${ }^{31}$ This study included 419 patients with metastatic or recurrent ESCC who were refractory or intolerant to previous fluoropyrimidinebased and platinum-based chemotherapy with either measurable or non-measurable lesions. After randomization, 210 patients were assigned to the nivolumab group and 209 to the chemotherapy group (Table 1).

Nivolumab monotherapy significantly improved OS compared to taxane (median OS 10.9 [95\% Cl 9.2-13.3] months versus 8.4 [95\% Cl 7.2-9.9] months, respectively; hazard ratio [HR] 0.77 [95\% $\mathrm{Cl} 0.62-0.96] ; \mathrm{p}=0.019$ ). The response rates for the nivolumab and chemotherapy groups were $19 \%(95 \% \mathrm{Cl} 14-26)$ and $22 \%(95 \% \mathrm{Cl} 15-29)$, and the median durations of response were 6.9 (95\% Cl: 5.4-11.1) months and 3.9 months $(95 \% \mathrm{Cl}$
2.8-4.2); the median durations for PFS were 1.7 months (95\% Cl 1.5-2.7) and 3.4 months $(95 \% \mathrm{Cl} 3.0-4.2)$, respectively. Additionally, there was no specific tendency of OS in the subgroup analysis based on PD-L1 expression evaluated by TPS. In the nivolumab group, the frequent treatment-related adverse events were rash (11.0\%), diarrhea (10.5\%), decreased appetite $(7.7 \%)$, and fatigue $(7.2 \%)$. Additionally, serious immune-related adverse events in the nivolumab group were diabetic ketoacidosis (one patient), intestinal lung disease (one patient), and pneumonitis (one patient).

The phase III ATTRACTION-3 phase III trial, which included previously treated patients with advanced ESCC, demonstrated that nivolumab was superior to taxane in terms of survival benefit and safety profile. ${ }^{31}$ Based on the results of the ATTRACTION-1 and ATTRACTION-3 trials, nivolumab was approved by the FDA on June 10, 2020 for patients with advanced esophageal cancer whose tumors are refractory to pyrimidine fluoride and platinum-based drugs.

\section{Nivolumab monotherapy as an adjuvant treatment for patients with esophageal cancer with resectable tumors}

The CheckMate 577 (ClinicalTrials.gov Identifier: NCT02743494) phase III trial was a randomized, multicenter, double-blind study that compared nivolumab monotherapy to placebo as the adjuvant treatment after complete resection of resectable esophageal cancer or esophagogastric junction tumors in patients who received neoadjuvant chemoradiation and in whom a pathologic complete response was not achieved. This trial demonstrated that nivolumab conferred significant survival benefits. ${ }^{33}$

In this trial, 794 patients were randomized to nivolumab ( $n=532$; nivolumab $240 \mathrm{mg}$, every 2 weeks for 16 weeks, followed by nivolumab $480 \mathrm{mg}$ every 4 weeks) or placebo ( $n=262)$. Approximately $70 \%$ of patients had esophageal adenocarcinoma and $30 \%$ of patients had ESCC. A pre-specified interim analysis showed that adjuvant nivolumab monotherapy conferred a statistically significant improvement in disease-free survival (DFS) when compared to placebo (median DFS 22.4 months [ $95 \% \mathrm{Cl}$ 6.6-34.0] versus 11.0 months [95\% Cl 8.3-14.3]; HR 0.69 [95\% Cl 0.56-0.86]; p=0.0003). Histological subgroup analysis showed that the median DFS of nivolumab-treated and placebo-treated patients with ESCC was 29.7 months and 11.0 months (HR 0.61 , respectively. This improvement was generally superior to that of the median DFS of patients with esophageal adenocarcinoma (19.4 months versus 11.1 months; HR 0.75). The most common grade $3 / 4$ treatment-related adverse events in the nivolumab group were pneumonitis $(0.8 \%)$ and rash $(0.8 \%)$; no treatment-related deaths were reported. ${ }^{33}$

The results of this trial suggest that adjuvant nivolumab monotherapy might be a new standard treatment for patients with esophageal cancer treated with neoadjuvant chemoradiotherapy followed by surgery that failed to achieve a pathological complete response.

\section{Other immune checkpoint inhibitors for patients with advanced esophageal cancer}

Pembrolizumab is a human monoclonal antibody that targets PD-1. The phase II KEYNOTE-180 trial study evaluated the efficacy and safety of pembrolizumab. The trial enrolled 121 patients; 63 (52\%) had ESCC and 58 (48\%) were PD-L1 positive. Tumors in patients were defined as PD-L1-positive if they had a CPS $\geq 10$ following immunohistochemical staining with $22 \mathrm{C} 3$ antibody. CPS was defined as the number of PD-L1 positive cells (tumor cells, macrophages, and lymphocytes) divided by the total number of tumor cells, multiplied by $100 .{ }^{34}$ 
Table 1: Clinical trials of nivolumab for metastatic or recurrent esophageal cancer

\begin{tabular}{|c|c|c|c|c|c|c|c|c|c|}
\hline Clinical trial & Phase & Line & Histology & $\begin{array}{l}\text { PD-L1 expression } \\
\text { antibody }\end{array}$ & $N$ & Regimen & $\begin{array}{l}\text { ORR } \\
(\%)\end{array}$ & $\begin{array}{l}\text { Median PFS } \\
\text { (month) }\end{array}$ & $\begin{array}{l}\text { Median OS } \\
\text { (month) }\end{array}$ \\
\hline ATTRACTION-130 & ॥ & $\geq 3$ & ESCC & $\begin{array}{l}\text { TPS } \\
28-8\end{array}$ & 65 & Nivolumab monotherapy & 17 & 1.5 & 10.8 \\
\hline ATTRACTION-331 & III & 2 & ESCC & $\begin{array}{l}\text { TPS } \\
28-8\end{array}$ & 419 & $\begin{array}{l}\text { Nivolumab monotherapy } \\
\text { versus chemotherapy }\end{array}$ & 19 & 1.7 & 10.9 \\
\hline CheckMate- $648^{32}$ & III & 1 & ESCC & $\begin{array}{l}\text { TPS } \\
28-8\end{array}$ & 939 & $\begin{array}{l}\text { Nivolumab + ipilimumab versus } \\
\text { CF + nivolumab versus CF }\end{array}$ & NA & NA & NA \\
\hline
\end{tabular}

$C F=$ cisplatin + 5-FU; ESCC = esophageal squamous cell carcinoma; $N A=$ not applicable; $O R R=$ objective response rate; $O S=$ overall survival; $P D-L 1=$ programmed death ligand 1; PFS = progression-free survival; TPS = tumor proportion score.

The primary endpoint of objective response rate was 9.9\% (95\% Cl 5.2-16.7) for all patients, 14.3\% (95\% Cl 6.7-25.4) for patients with ESCC, 5.3 (95\% Cl 1.114.4) for patients with esophageal adenocarcinoma, $13.8 \%(95 \% \mathrm{Cl} 6.1-25.4)$ for patients with $\mathrm{CPS} \geq 10$, and $6.3 \%$ (95\% $\mathrm{Cl} 1.8-15.5$ ) in $\mathrm{CPS}<10$; the median duration of response was not reached. In this trial, frequent treatment-related grade $3 / 4$ adverse events were type 1 diabetes (3\%), and pneumonia (2\%). Pneumonia was the single grade 5 treatment-related adverse event. ${ }^{34}$

After the promising results of the KEYNOTE-180 trial, the phase III KEYNOTE-181 trial compared pembrolizumab monotherapy (200 mg every 3 weeks) with chemotherapy (paclitaxel 80-100 mg/m² on days 1, 8, 15, every 4 weeks; docetaxel $75 \mathrm{mg} / \mathrm{m}^{2}$, every 3 weeks; or irinotecan $180 \mathrm{mg} / \mathrm{m}^{2}$, every 2 weeks) as second-line treatment for metastatic or recurrent esophageal cancer. The primary endpoints were OS in all patients, OS for patients with PD-L1 CPS $\geq 10$, and OS for patients with ESCC. In this trial, 628 patients were randomized $1: 1$ to pembrolizumab or an investigator-selected regimen. Compared with chemotherapy, treatment pembrolizumab monotherapy of 222 patients with esophageal cancer with PD-L1 CPS $\geq 10$ improved OS (median OS 9.3 months [95\% Cl 6.6-12.5] versus 6.7 months [95\% Cl 5.1-8.2]; HR 0.69 [95\% $\mathrm{Cl} 0.52-0.93] \mathrm{p}=0.0074)$. On the other hand, pembrolizumab monotherapy did not improve OS in 401 patients with ESCC (median OS 8.2 months [95\% $\mathrm{Cl}$ 6.7-10.3] versus 7.1 months [95\% $\mathrm{Cl} 6.1-8.2$ ]; HR 0.78 [95\% $\mathrm{Cl} 0.63-0.96$ ]; $\mathrm{p}=0.0095$ ) or in all 628 patients (median OS 7.1 months [95\% $\mathrm{Cl} 6.2-8.1$ ] versus 7.1 months [95\% Cl 6.3-8.0]; HR 0.89 [95\% Cl 0.75-1.05], $p=0.056$ ). ${ }^{35.36}$

Based on the results of these two KEYNOTE trials, the FDA approved pembrolizumab in 2019 specifically for the treatment of patients with advanced ESCC with PD-L1-positive tumors (CPS $\geq 10$ ) and in whom disease has progressed following at least one previous systemic treatment.

KEYNOTE-590 was a randomized, blinded, phase III trial that compared pembrolizumab (200 mg every 3 weeks for $\leq 35$ cycles) plus chemotherapy (5-FU $800 \mathrm{mg} / \mathrm{m}^{2}$ for days $1-5$ every 3 weeks for $\leq 35$ cycles + cisplatin 80 $\mathrm{mg} / \mathrm{m}^{2}$ every 3 weeks for $\leq 6$ cycles) with chemotherapy alone (placebo + 5 -FU $800 \mathrm{mg} / \mathrm{m}^{2}$ for days $1-5$ every 3 weeks for $\leq 35$ cycles + cisplatin 80 $\mathrm{mg} / \mathrm{m}^{2}$ every 3 weeks for $\leq 6$ cycles) for metastatic or recurrent esophageal adenocarcinoma, ESCC, and esophagogastric junction cancer. This trial randomized 274 (73.5\%) patients with ESCC to pembrolizumab plus chemotherapy and 274 (72.9\%) patients with ESCC to chemotherapy. ${ }^{37}$

Results of KEYNOTE-590 showed that pembrolizumab plus doublet chemotherapy was superior to doublet chemotherapy in terms of OS and PFS. The median OS in patients with ESCC with CPS $\geq 10$ was 13.9 months (95\% Cl 11.1-11.7) versus 8.8 months (95\% Cl 7.8-10.5) (HR $0.57[95 \% \mathrm{Cl}$
0.43-0.75]; $p<0.0001)$, in patients with ESCC was 12.6 months $(95 \% \mathrm{Cl}$ 10.2-14.3) versus 9.8 months (95\% Cl 8.6-11.1) (HR 0.72 [95\% Cl 0.6-0.88]; $\mathrm{p}<0.0006$ ), in patients with $\mathrm{CPS} \geq 10$ was 13.5 months ( $95 \% \mathrm{Cl} 11.1-15.6$ ) versus 9.4 months (95\% Cl 8.0-10.7) (HR 0.62 [95\% Cl 0.49-0.78]; $p<0.0001$ ), and in all patients was $12.4(95 \% \mathrm{Cl} 10.5-14.0)$ months versus 9.8 months (95\% Cl 8.8-10.8) (HR 0.73 [95\% Cl 0.62-0.86]; $p<0.0001)$. The median PFS in patients with ESCC was 6.3 months $(95 \% \mathrm{Cl} 6.2-6.9)$ versus 5.8 months (95\% Cl 5.0-6.1) (HR 0.65 [95\% Cl 0.54-0.78]; $\mathrm{p}<0.0001)$, in patients with CPS $\geq 10$ was 7.5 months $(95 \% \mathrm{Cl} 6.2-8.2)$ versus 5.5 months $(95 \% \mathrm{Cl}$ 4.3-6.0) (HR 0.51 [95\% Cl 0.41-0.65]; $\mathrm{p}<0.0001$ ), and in all patients was 6.3 months (95\% $\mathrm{Cl} 6.2-6.9)$ versus 5.8 months (95\% Cl 5.0-6.0) (HR 0.65 [95\% $\mathrm{Cl} 0.55-0.76] ; p<0.0001)$. The secondary endpoint of objective response rate was also significantly improved $(45 \%$ [95\% $\mathrm{Cl} 39.9-50.2]$ versus $29.3 \%$ [95\% Cl 24.7-34.1]; $p<0.0001)$. Immune-related grade 3 or higher adverse events were reported in $7 \%$ of patients in the pembrolizumab plus doublet chemotherapy group. ${ }^{37}$ The results of the KEYNOTE-590 trial suggest that pembrolizumab plus doublet chemotherapy could be a new standard first-line treatment in patients with esophageal cancer with metastatic or recurrent disease.

Camrelizumab is a fully-humanized, selective IgG4-к monoclonal antibody against PD-1. The ESCORT trial was a randomized, open-label, phase III study conducted at 43 centers in China. ${ }^{38}$ In this trial, 457 patients with ESCC were randomized to either camrelizumab $(n=229)$ or chemotherapy $(n=228)$. Median OS was 8.3 months (95\% $\mathrm{Cl} 6.8-9.7$ ) in the camrelizumab group versus 6.2 months (95\% Cl 5.7-6.9) in the chemotherapy group (HR 0.71 [95\% Cl 0.57-0.87], two-sided $p=0.0010$ ), demonstrating that camrelizumab monotherapy was superior to chemotherapy when used as a second-line treatment. In the camrelizumab group, frequent grade 3 or higher adverse events were anemia (3\%) and liver dysfunction (2\%), and there were seven camrelizumab-related deaths (three unknown causes, one enterocolitis, one liver dysfunction, one pneumonitis, and one myocarditis). Therefore, camrelizumab may become a standard option for second-line treatment for patients with ESCC in China. ${ }^{38}$

\section{Ongoing clinical trials of immune checkpoint inhibitors for patients with esophageal cancer}

There are several ongoing phase III trials that are focused on patients with metastatic or recurrent esophageal cancer. The phase III CheckMate 648 trial, to compare nivolumab plus doublet chemotherapy or nivolumab plus ipilimumab with doublet chemotherapy as a first-line treatment for untreated patients with metastatic or recurrent ESCC (ClinicalTrails.gov Identifier: NCT03143153), ${ }^{32}$ has finished recruiting and is awaiting analysis. Tislelizumab (BGB-A317) is an anti-PD-1 antibody that was developed for 
Table 2: Clinical trials of nivolumab for locally advanced esophageal cancer

\begin{tabular}{|c|c|c|c|c|c|c|c|c|}
\hline Clinical trial & Phase & Histology & $\begin{array}{l}\text { PD-L1 expression } \\
\text { antibody }\end{array}$ & $\mathrm{N}$ & Regimen & $\begin{array}{l}\mathrm{PCR} \\
(\%)\end{array}$ & $\begin{array}{l}\text { Median DFS } \\
\text { (month) }\end{array}$ & $\begin{array}{l}\text { Median OS } \\
\text { (month) }\end{array}$ \\
\hline $\begin{array}{l}\text { NOBEL } \\
\text { (UMIN000035889) }\end{array}$ & $\|$ & ESCC & NA & Recruiting & dCRT + sequential nivolumab & NA & NA & NA \\
\hline $\begin{array}{l}\text { CRUCIAL } \\
\text { (NCT03437200) }\end{array}$ & ॥ & ESCC, EAC & NA & Recruiting & $\begin{array}{l}\text { dCRT + concurrent and sequential nivolumab } \\
\text { or nivolumab + ipilimumab }\end{array}$ & NA & NA & NA \\
\hline $\begin{array}{l}\text { NCT03044613 } \\
(\operatorname{arm~A)~})^{41}\end{array}$ & 1 & ESCC, EAC & NA & 16 & $\begin{array}{l}\text { Induction nivolumab } \\
+ \text { preoperative CRT + concurrent nivolumab }\end{array}$ & 40 & NA & NA \\
\hline $\begin{array}{l}\text { CheckMate } 577 \\
\text { (NCT02743494) }\end{array}$ & III & ESCC, EAC & $\begin{array}{l}\text { TPS } \\
28-8\end{array}$ & 794 & $\begin{array}{l}\text { Preoperative CRT } \\
\pm \text { postoperative nivolumab }\end{array}$ & NA & 22.4 & NA \\
\hline $\begin{array}{l}\text { FRONTIER } \\
\text { (NCT03914443) }\end{array}$ & 1 & ESCC & $\begin{array}{l}\text { TPS } \\
28-8\end{array}$ & Recruiting & $\begin{array}{l}\text { Preoperative CF + nivolumab or preoperative } \\
\text { DCF + nivolumab }\end{array}$ & NA & NA & NA \\
\hline
\end{tabular}

$C F=$ cisplatin $+5-F U ; C R T=$ chemoradiotherapy; $D C F=$ docetaxel + cisplatin $+5-F U ; d C R T=$ definitive chemoradiotherapy; $D F S=$ disease-free survival; $E A C=$ esophageal adenocarcinoma; $E S C C=$ esophageal squamous cell carcinoma; $N A=$ not applicable; OS = overall survival; $p C R=$ pathological complete response rate; $P D-L 1=$ programmed death ligand 1; TPS = tumor proportion score.

the treatment of patients with metastatic or recurrent ESCC in China. The phase III RATIONALE-302 trial (ClinicalTrails.gov Identifier: NCT03430843) is a randomized study comparing the efficacy of tislelizumab with chemotherapy (taxane or irinotecan) as second-line treatment in patients with metastatic or recurrent ESCC. This trial has also finished recruiting and is awaiting analysis. In addition, the phase III RATIONALE-306 trial, to compare tislelizumab plus chemotherapy (5-FU plus platinum or paclitaxel plus platinum) with chemotherapy as a first-line treatment for untreated patients with metastatic or recurrent ESCC, is recruiting (ClinicalTrails.gov Identifier: NCT03783442).

Based on the results of the phase III PACIFIC trial, ${ }^{39}$ there are ongoing phase II and phase III trials in patients with unresectable, locally advanced esophageal cancer to evaluate the efficacy of definitive chemoradiation followed by an immune checkpoint inhibitor. The phase II NOBEL trial will evaluate the efficacy and safety of 5-FU- and cisplatinbased definitive chemoradiation plus nivolumab followed by sequential nivolumab monotherapy (UMIN ID: UMIN000035889) (Table 2), ${ }_{10,41}$ and the phase II TENERGY trial will evaluate the efficacy and safety of definitive chemoradiation followed by sequential atezolizumab (anti-PD-L1 antibody) monotherapy (UMIN ID: UMIN000034373). ${ }^{40}$ For inoperable, locally advanced esophageal cancer, patients are being recruited to the phase II CRUCIAL trial to evaluate efficacy and safety of FOLFOX-based definitive chemoradiation plus nivolumab followed by sequential nivolumab monotherapy, and chemoradiation plus nivolumab and ipilimumab followed by sequential nivolumab and ipilimumab (ClinicalTrails.gov Identifier: NCT03437200) (Table 2). Patients are also being recruited to the phase III KEYNOTE-975 trial that will evaluate the efficacy and safety of 5-FU- and cisplatin- or FOLFOXbased chemoradiation plus concurrent pembrolizumab monotherapy (ClinicalTrails.gov Identifier: NCT04210115).

Finally, some phase I and phase II trials that focused on patients with resectable, locally advanced esophageal cancer have now reported their findings. A phase I trial to evaluate the safety and efficacy of induction nivolumab monotherapy and neoadjuvant chemoradiation plus nivolumab followed by surgery for patients with resectable esophageal cancer or gastroesophageal cancer has been conducted. In 16 patients, treatmentrelated adverse events occurred in 12 patients (75.0\%), and 4 patients (25.0\%) showed grade 3 adverse events (dyspnea and upper respiratory tract infection, transaminitis, and rash). Fourteen patients (87.5\%) received all planned doses of nivolumab. The pathological complete response rate was $28.6 \%$ (4/14) for patients with esophageal adenocarcinoma, and 50.0\% (1/2) for patients with ESCC. ${ }^{41}$ A phase II trial to evaluate the efficacy and safety of neoadjuvant chemoradiation plus pembrolizumab followed by surgery and adjuvant pembrolizumab monotherapy for patients with resectable ESCC (clinical T1N1-2M0, clinical T2-4aNO-2M0) has been conducted. The pathological complete response rate was $23.1 \%(6 / 26)$. In all 28 patients, 3 died during the preoperative period (massive hemorrhage) and after surgery (acute lung injury). ${ }^{43}$

The phase II PERFECT trial evaluated the efficacy and safety of neoadjuvant chemoradiation plus atezolizumab followed by surgery for patients with resectable esophageal adenocarcinoma. The completion ratio was $75 \%$ (30/40) and the pathological complete response was 37\% (11/30). Immunerelated adverse events of grade 3 rash (5\%), grade 2 colitis (5\%), and thyroiditis (5\%) were reported. ${ }^{44} \mathrm{~A}$ phase $\mathrm{I} / \mathrm{II}$ trial has also evaluated the efficacy and safety of preoperative chemoradiation plus avelumab (anti-PD-L1 antibody) followed by surgery and postoperative avelumab monotherapy for patients with resectable locally advanced esophageal cancer (clinical T1N1M0, clinical T2-3N0-2M0). The pathological complete response was $43 \%(3 / 7)$. Grade 2 hypothyroidism (5\%) was the only immune-related adverse event reported. ${ }^{45}$ The above trials are developing treatments based on neoadjuvant chemoradiation. However, there is also an ongoing a phase I trial based on neoadjuvant chemotherapy (JCOG1804E/FRONTiER) that will evaluate the safety and efficacy of nivolumab plus 5-FU and cisplatin or nivolumab plus docetaxel and 5-FU, cisplatin for patients with resectable, locally advanced ESCC (ClinicalTrails.gov Identifier: NCT03914443)² (Table 2).

\section{Efficacy of immune checkpoint inhibitors by histology}

The current article focused on the efficacy of nivolumab for patients with ESCC, the efficacy of immune checkpoint inhibitors might be different between ESCC and esophageal adenocarcinoma. In the ATTRACTION-130 and ATTRACTION-3 trials, ${ }^{31}$ only patients with ESCC were included. In the ATTRACTION-3 trial, OS for patients with ESCC was reported at HR 0.77 (95\% $\mathrm{Cl}$ 0.62-0.96). ${ }^{31}$ On the other hand, in the KEYNOTE-180 and KEYNOTE-181 trials, patients with ESCC and those with esophageal adenocarcinoma were included. In the subgroup analysis of the KEYNOTE-181 trial, OS for patients 
with ESCC and esophageal adenocarcinoma was reported at HR $0.77(95 \% \mathrm{Cl}$ 0.63-0.96) and HR 1.12 (95\% Cl 0.85-1.47), respectively; and in the subgroup analysis of the KEYNOTE-590 trial, ${ }^{37}$ OS for patients with ESCC and esophageal adenocarcinoma patients were reported at $\mathrm{HR} 0.72(95 \% \mathrm{Cl} 0.60-0.88)$ and HR 0.74 (95\% Cl 0.54-1.02), respectively. Additionally, in a subgroup analysis of the CheckMate 577 trial, ${ }^{33} \mathrm{HR}$ for DFS in patients with ESCC and esophageal adenocarcinoma were reported at 0.61 and 0.75 , respectively.

In consideration of these data, the efficacies of immune checkpoint inhibitors tend to be numerically lower in adenocarcinoma compared to ESCC, regardless of palliative and adjuvant treatments; however, at present, the difference is unclear. The biological mechanism of histological difference in response to immune checkpoint inhibitors has not yet been revealed. Therefore, further biological studies are needed to select appropriate subgroups for immune checkpoint inhibitors and to overcome tolerance for immune checkpoint inhibitors.

\section{Conclusion and future perspective}

Based on the results of the ATTRACTION-3 trial, nivolumab became the standard option for second-line treatment of patients with metastatic and recurrent ESCC regardless of PD-L1 expression. ${ }^{31}$ On the other hand, based on the results of the KEYNOTE-181 trial, pembrolizumab has also been adopted as the second-line treatment for patients with metastatic or recurrent ESCC with CPS $\geq 10.35,36$ Given access to second-line treatment for patients with advanced ESCC, nivolumab might be easier to administrate than pembrolizumab. However, the KEYNOTE-590 trial showed superiority of pembrolizumab plus doublet chemotherapy compared with doublet chemotherapy as first-line treatment for metastatic or recurrent patients with esophageal cancer. ${ }^{37}$ Therefore, pembrolizumab might be used more frequently as a first-line treatment and nivolumab monotherapy might be used less frequently as a second-line treatment as long as there is no evidence of immune checkpoint inhibitor continuation beyond progression. However, depending on the results of the checkMate 648 trial, nivolumab plus ipilimumab or nivolumab plus doublet chemotherapy might be one of the standard treatments (ClinicalTrails.gov Identifier: NCT03143153). ${ }^{32}$

On the other hand, adjuvant nivolumab monotherapy may be used more frequently for patients with resectable esophageal cancer who received neoadjuvant chemoradiation followed by surgery, and those in whom a complete response was not achieved. In addition to neoadjuvant chemoradiation followed by surgery, neoadjuvant chemotherapy followed by surgery is increasingly used as a standard treatment. Therefore, further research into the efficacy of immune checkpoint inhibitors in the context of preoperative chemotherapy and surgery is required.]
1. Bray F, Ferlay J, Soerjomataram I, et al. Global cancer statistics 2018 GLOBOCAN estimates of incidence and mortality worldwide for 36 cancers in 185 countries. CA Cancer J Clin. 2018; 68: 394-424.

2. Abnet $\mathrm{CC}$, Arnold M, Wei WQ. Epidemiology of esophageal squamous cell carcinoma. Gastroenterology. 2018:154:360-73.

3. Niei B, MCCarty TR, Birk JW. Trends in esophageal cancer survival in United States adults from 1973 to 2009: a SEER database analysis. J Gastroenterol Hepatol. 2016;31:1141-6.

4. Torre LA, Siegel RL, Ward EM, Jemal A. Global cancer incidence and mortality rates and trends--an update. Cancer Epidemiol Biomarkers Prev. 2016;25:16-27.

5. Murphy G, McCormack V, Abedi-Ardekani B, et al. International cancer seminars: a focus on esophageal squamous cell carcinoma. Ann Oncol. 2017:28:2086-93.

6. Rustgi AK, El-Serag HB. Esophageal carcinoma. N Eng/ J Med. 2014:371:2499-509.

7. NCCN Clinical Practice Guidelines in Oncology (NCCN Guidelines ${ }^{\circledR}$ ) Esophageal and Esophagogastric Junction Cancers Version 4.2020. Available at: www.nccn.org/professionals/physician_gls/pdf/ esophageal.pdf (accessed December 19, 2020).

8. Lordick F, Mariette C, Haustermans K, et al. Oesophageal cancer: ESMO clinical practice guidelines for diagnosis, treatment and follow-up. Ann Oncol. 2016;27:v50-7.

9. Ford $\mathrm{HE}$, Marshall $\mathrm{A}$, Bridgewater JA, et al. Docetaxel versus active symptom control for refractory oesophagogastric adenocarcinoma (COUGAR-02): an open-label, phase 3 randomised controlled trial. Lancet Oncol. 2014;15:78-86.

10. Kato K, Tahara M, Hironaka S, et al. A phase II study of paclitaxel by weekly 1-h infusion for advanced or recurrent esophageal cancer in patients who had previously received platinum-based chemotherapy. Cancer Chemother Pharmacol. 2011;67:1265-72.

11. Muro K, Hamaguchi T, Ohtsu A, et al. A phase II study of single-agent docetaxel in patients with metastatic esophageal cancer. Ann Oncol. 2004;15:955-9.

12. Boussiotis VA. molecular and biochemical aspects of the PDcheckpoint pathway. N Eng/ J Med. 2016;375:1767-78

13. Raufi AG, Klempner SJ. Immunotherapy for advanced gastric and esophageal cancer. preclinical rationale and ongoing clinica investigations. J Gastrointest Oncol. 2015;6:561-9.

14. Pardoll DM. The blockade of immune checkpoints in cancer immunotherapy. Nat Rev Cancer. 2012;12:252-64.

15. Topalian SL, Hodi FS, Brahmer JR, et al. Safety, activity, and immune correlates of anti-PD-1 antibody in cancer. N Eng/ J Med. 2012;366:2443-54

16. Brahmer J, Reckamp KL, Baas $\mathrm{P}$, et al. Nivolumab versus docetaxel in advanced squamous-cell non-small-cell lung cancer. N Eng/ I Med. 2015:373:123-35.

17. Ansell SM, Lesokhin AM, Borrello I, et al. PD-1 blockade with nivolumab in relapsed or refractory Hodgkin's lymphoma. N Eng/ J Med. 2015;372:311-9.

18. Motzer RJ, Escudier B, McDermott DF, et al. Nivolumab versus everolimus in advanced renal-cell carcinoma. N Eng/ J Med. 2015;373:1803-13.
19. Ferris RL, Blumenschein $\mathrm{G} \mathrm{Jr}$, Fayette J, et al. Nivolumab for recurrent squamous-cell carcinoma of the head and neck. NEngI I Med. 2016;375:1856-67.

20. Sharma $P$, Retz $M$, Siefker-Radtke $A$, et al. Nivolumab in metastatic urothelial carcinoma after platinum therapy (CheckMate 275): a multicentre, single-arm, phase 2 trial. Lancet Oncol. 2017:18:312-22.

21. Overman MJ, McDermott R, Leach JL, et al. Nivolumab in patients with metastatic DNA mismatch repair-deficient or microsatellite instability-high colorectal cancer (CheckMate 142): an open-label, multicentre, phase 2 study. Lancet Oncol. 2017;18:1182-91.

22. El-Khoueiry AB, Sangro B, Yau T, et al. Nivolumab in patients with advanced hepatocellular carcinoma (CheckMate 040): an open-labe, non-comparative, phase $1 / 2$ dose escalation and expansion trial. Lancet. 2017:389:2492-502.

23. Kang YK, Boku N, Satoh T, et al. Nivolumab in patients with advanced gastric or gastro-oesophageal junction cancer refractory to or intolerant of, at least two previous chemotherapy regimens (ONO-4538-12, ATTRACTION-2): a randomised, double-blind, placebocontrolled, phase 3 trial. Lancet. 2017:390:2461-71.

24. Okada M, Kijima T, Aoe K, et al. Clinical efficacy and safety of nivolumab: results of a multicenter, open-label, single-arm, Japanese phase II study in malignant pleural mesothelioma (MERIT). Clin Cancer Res. 2019:25:5485-92.

25. OPDIVO (nivolumab). Highlights of prescribing information, 2018. Available at: www.accessdata.fda.gov/drugsatfda_docs/ label/2019/125554s070lbl.pdf (accessed December 19, 2020).

26. Postow MA, Chesney J, Pavlick AC, et al. Nivolumab and ipilimumab versus ipilimumab in untreated melanoma. N Eng/ I Med. 2015;372:2006-17.

27. Chen $\mathrm{K}$, Cheng $\mathrm{G}$, Zhang $\mathrm{F}$, et al. Prognostic significance of programmed death-1 and programmed death-ligand 1 expression in patients with esophageal squamous cell carcinoma. Oncotarget. 2016;7:30772-80.

28. Guo W, Wang P, Li N, et al. Prognostic value of PD-L1 in esophagea squamous cell carcinoma: a meta-analysis. Oncotarget. 2017;9:13920-33.

29. Tsao MS, Kerr KM, et al. PD-L1 immunohistochemistry comparability study in real-life clinical samples: Results of Blueprint Phase 2 Project. I Thorac Oncol. 2018:13:1302-11.

30. Kudo T, Hamamoto Y, Kato K, et al. Nivolumab treatment for oesophageal squamous-cell carcinoma: an open-label, multicentre, phase 2 trial. Lancet Oncol. 2017;18:631-9.

31. Kato $K$, Cho BC, Takahashi M, et al. Nivolumab versus chemotherapy in patients with advanced oesophageal squamous cell carcinoma refractory or intolerant to previous chemotherapy (ATTRACTION-3): a multicentre, randomised, open-label, phase 3 trial. Lancet Oncol. 2019:20:1506-17.

32. Ajani JA, Kato K, Doki Y, et al. CheckMate 648: a randomized phase 3 study of nivolumab plus ipilimumab or nivolumab combined with fluorouracil plus cisplatin versus fluorouracil plus cisplatin in patients with unresectable advanced, recurrent, or metastatic previously untreated esophageal squamous cell carcinoma. J Clin Oncol. 2018;36(Suppl. 4):TPS193.
33. Kelly RJ, Ajani JA, Kuzdzai J, et al. Adjuvant nivolumab in resected esophageal or gastroesophageal junction cancer (EC/GEJC) following neoadjuvant chemoradiation therapy (CRT): first results of the CheckMate 577 study. Presented at ESMO Virtual Congress, September 21, 2020.

34. Shah MA, Kojima T, Hochhauser D, et al. Efficacy and safety of pembrolizumab for heavily pretreated patients with advanced, metastatic adenocarcinoma or squamous cell carcinoma of the esophagus: the phase 2 KEYNOTE-180 study. JAMA Oncol. 2019;5:546-50.

35. Kojima T, Muro K, Francois E, et al. Pembrolizumab versus chemotherapy as second-line therapy for advanced esophageal cancer. phase III KEYNOTE-181 study. J Clin Oncol. 2019;37(Suppl. 15): 4010.

36. Kojima T, Shah MA, Muro K, et al. Randomized phase III KEYNOTE-181 study of pembrolizumab versus chemotherapy in advanced esophageal cancer. J Clin Oncol. 2020;38:4138-48.

37. Kato K, Sun JM, Shah MA, et al. Pembrolizumab plus chemotherapy versus chemotherapy as first-line therapy in patients with advanced esophageal cancer: the phase 3 KEYNOTE-590 study. Presented at ESMO Virtual Congress, September 21, 2020

38. Huang J, Xu J, Chen Y, et al. Camrelizumab versus investigator's choice of chemotherapy as second-line therapy for advanced or metastatic oesophageal squamous cell carcinoma (ESCORT): a multicentre, randomised, open-label, phase 3 study. Lancet Oncol. 2020;21:832-42.

39. Antonia SJ, Villegas A, Daniel D, et al. Overall survival with durvalumab after chemoradiotherapy in stage III NSCLC. N Eng/ I Med. 2018;379:2342-50

40. Bando H, Kotani D, Tsushima T, et al. TENERGY: multicenter phase II study of atezolizumab monotherapy following definitive chemoradiotherapy with 5-FU plus cisplatin in patients with unresectable locally advanced esophageal squamous cell carcinoma. BMC Cancer. 2020;20:336

41. Kelly RJ, Smith KN, Anagnostou V, et al. Neoadjuvant nivolumab plus concurrent chemoradiation in stage $\|/\| / \|$ esophageal/gastroesophageal junction cancer. J Clin Oncol. 2019;37(Suppl. 4):142.

42. Yamamoto S, Kato K, Daiko H, et al. Feasibility study of nivolumab as neoadjuvant chemotherapy for locally esophageal carcinoma: FRONTIER (JCOG1804E). Future Oncol. 2020;16:1351-7.

43. Hong MH, Kim HR, Park SY, et al. A phase II trial of preoperative chemoradiotherapy and pembrolizumab for locally advanced esophageal squamous cell carcinoma (ESCC). J Clin Oncol. 2019;37(Suppl. 15):4027.

44. van den Ende T, de Clercq NC, van Berge Henegouwen Ml, et al. A phase II feasibility trial of neoadjuvant chemoradiotherapy combined with atezolizumab for resectable esophageal adenocarcinoma: the PERFECT trial. J Clin Oncol. 2019;37(Suppl. 5):4045.

45. Uboha NV, Maloney JD, MCCarthy D, et al. Safety of neoadjuvant chemoradiation (CRT) in combination with avelumab (A) in the treatment of resectable esophageal and gastroesophageal junction (E/GEJ) cancer. J Clin Oncol. 2019;37(Suppl. 15):4041. 